\title{
Preparing for Service-Oriented Computing: A Composite Design Pattern for Stubless Web Service Invocation
}

\author{
Paul A. Buhler ${ }^{1}$, Christopher Starr', William H. Schroder ${ }^{1}$, and José M. Vidal ${ }^{2}$ \\ ${ }^{1}$ College of Charleston, Dept. of Computer Science, \\ 66 George Street, Charleston, SC 29424, USA \\ \{buhlerp, starrc, schroderw\} @cofc.edu \\ ${ }^{2}$ University of South Carolina, Computer Science and Engineering \\ Columbia, SC 29208, USA \\ vidal@sc. edu
}

\begin{abstract}
The ability to dynamically bind to Web services at runtime is becoming increasingly important as the era of Service-Oriented Computing (SOC) emerges. With SOC selection and invocation of Web service partners will occur in software at run-time, rather than by software developers at design and compile time. Unfortunately, the marketplace has yet to yield a predominate applications programming interface for the invocation of Web services. This results in software that is deeply ingrained with vendor-specific calls, which is problematic because Web service technology is changing at a rapid pace. In order to leverage the latest developments, code often needs to be heavily refactored to account for changing invocation interfaces. This paper explores the mitigation of this problem through the application of software design patterns.
\end{abstract}

\section{Introduction}

Web services will be the foundational technology that will underpin future distributed, Internet-based computing systems as the worlds of Service-Oriented Computing (SOC), Multiagent Systems (MAS), and Business Process Management (BPM) converge. Software toolkits, which build on open standards such as HTTP, SOAP, WSDL and UDDI, allow developers to compose web services into robust business applications by harnessing the power standards. Unfortunately, the marketplace has yet to yield a predominate toolkit represented by a standard Applications Programming Interface (API) for dynamic, fully stubless invocation of Web services.

The lack of a standard API results in software that is deeply ingrained with vendorspecific API's. However, because Web service technology is changing at a rapid pace with new tools and techniques frequently becoming available, code often needs to be significantly refactored to account for changing interfaces. To compensate for this problem, we have used software engineering principles and software design patterns to create a composite pattern, which insulates Web service client code from the peculiarities of any specific vendor interface. This framework approach enhances code 
stability while providing the flexibility to experiment with various approaches to the dynamic invocation of Web services.

Just as Web services are components intended to be composed, there is a growing realization that design patterns can be composed or aggregated into larger units. As early as 1997, it was shown that composite patterns could possess a set of characteristics that exceeded those of the individual contributing patterns [4]. The notion that patterns and pattern compositions can be used as premier design components for design creation, changes the design approach by introducing larger-grained design components. This approach is used in the Pattern-Oriented Analysis and Design (POAD) technique [5]. The pattern concept has been applied with coarser granularity at the architectural level as architectural patterns [1] and the application domain level as framework patterns [3].

To isolate the application interface from its implementation, a Composite Pattern for Web service Invocation was constructed using a composition of design patterns. To achieve the desired level of flexibility and extensibility for the framework, the overall class structure of the design is provided by the Bridge pattern. The decoupling avoids a permanent binding between an abstraction and its implementation allowing the implementation to be selected or switched at runtime. Additional flexibility can be derived by introducing the Factory Method pattern. The Factory Method is a creational design pattern, which defers the instantiation of a product to subclasses localized external to the client application [2]. The pattern has applicability when the client cannot anticipate the class of objects it needs until runtime.

The composition of the Factory Method and the Bridge provide a combination of design assets which decouples clients from the concrete implementations, enabling the choice of implementation to be made based upon some runtime condition. The resulting composite pattern of the Bridge and Factory Method patterns provides an agile software design for the decoupling of the invocation services that are found and bound at run-time.

\section{References}

[1] Buschmann, F. Pattern-oriented software architecture : a system of patterns. Wiley, Chichester ; New York, 1996.

[2] Gamma, E. Design patterns : elements of reusable object-oriented software. AddisonWesley, Reading, Mass., 1995.

[3] Johnson, R.E. Documenting frameworks using patterns. In Proceedings of the Conference on Object-Oriented Programming Systems, Languages, and Applications (OOPSLA), ACM Press, 63-70, 1992.

[4] Riehle, D. Composite Design Patterns. In Proceedgins of the Conference on ObjectOriented Programming, Systems, Languages, and Applications (OOPSLA), ACM Press, 218-228, 1997.

[5] Yacoub, S.M. and Ammar, H.H. Pattern oriented analysis and design : composing patterns to design software systems. Addison-Wesley, Boston, MA, 2004. 